\title{
The Measured Equation of Invariance A New Concept in Field Computation
}

\author{
Kenneth K. Mei * Rafael Pous, Zhaoqing Chen, and Yao Wu Liu
}

\section{Abstract}

Computations of electromagnetic fields are based either on differential equations or on integral equations. The differential equation approach using finite difference (FD) or finite element (FE) methods result in sparse matrices, which is an advantage, but have to cover large volumes, which is a disadvantage. The integral equation approach using the method of moments (MOM) limits the mesh to the surface of the object, which is an advantage, but results in full matrices, which is a disadvantage. The ideal case would be to reduce the finite difference type equations close to the object surface and still preserve the sparsity of the matrices. The measured equation of invariance (MEI) is a new concept in field computation capable of approaching this ideal situation. The mathematics and reasonings to reach a new computational method based on this new concept will be presented. It is shown that the method is robust for both convex and concave objects, much faster than the MOM, and uses a fraction of the memory.

The radiation conditions, or absorbing boundary conditions, are physical conditions imposed on the outer mesh boundaries of finite difference or finite element meshes in lieu of the differential equations in order to terminate the mesh [1], [2]. Since memory and computation time both grow very fast with the mesh volume, there have been efforts to bring the absorbing boundary close to the object boundary [3], [4]. But, those close-tothe-object-surface absorbing boundary conditions give mixed results, and in general are not robust.

We observe that the conventional finite equation is actually a general equation of invariance, in that any solution of the differential equation satisfies the $\mathrm{FD}$ equation to second order. For example, let N-point FD equation be

$$
\sum_{i=1}^{N} c_{i} \cdot u_{i}=0
$$

and $\phi(\bar{r})$ be a solution of the differential equation. Then, substituting $\phi\left(\bar{r}_{i}\right)$ in place of $u_{i}$ we get

$$
\sum_{i=1}^{N} c_{i} \cdot \phi\left(\bar{r}_{i}\right)=o\left(h^{2}\right)
$$

*This research is supported by California MICRO program, and the industrial sponsor Hughes Aircraft Co. under contract No. $442427-57465$ 
where $h$ is the mesh size as shown in Fig. 1. Conversely, if $N-1$ different solutions of the differential equation $\left.\phi^{(} k\right)(\bar{r}), k=1,2, \ldots, N-1$, are available, we are able to find the coefficients $c_{i}$ without using the finite difference approach.

The coefficients $c_{i}$ are not unique, and by choosing various $\left.\phi^{(} k\right)(\bar{r})$ as measures, we may find finite difference type equations, which are directional, that is, the equation allows certain solutions and rejects the rest.

There are two types of measures. Those which are object independent, and those which are object dependent. The absorbing boundary conditions are in general object independent, i.e., the equations so derived are the same regardless of the object geometry. In this paper, we make use of measures which are object dependent. The measures are produced by a set of currents, which we call metrons, on the object surface. For an $\mathrm{N}$-point finite difference equation $N-1$ metrons will be required. The object dependent measured equation of invariance is not an absorbing boundary condition, because it also allows incoming waves. Therefore, the method can also be used to solve scattering by concave objects.

Figures 2 and 3 show the electric currents induced on the surface of a square cylinder by an incident wave for the TE and TM cases respectively. In all four calculations, the result agrees very closely with the one obtained using the Method of Moments (MOM). Figures 4 and 5 show the same type of computation but for a concave cylinder. Again, very good agreement is shown with MOM results. The MEI method can be used very near the surface of concave objects because it is not a radiation boundary condition, but an object-dependent near-field condition. In Fig. 6 the CPU times for the two methods are compared. The greater slope of the logarithmic graph indicates that the MOM is of a greater order than the MEI method.

Extensive testing of the method has been done in two dimensions, and the method has proven to be robust and give stable results as long as the mesh spacing is small in terms of the wavelength.

\section{References}

(1) B. Engquist and A. Majda, "Absorbing Boundary Conditions for the Numerical Simulation of Waves," Math. Comp., vol 31, pp. 629-651, 1977.

[2] A. Bayliss and E. Turkel, "Radiation Boundary Conditions for Wave-like Equations," Pure and Appl. Math., vol 33, pp. 707-725, 1980.

[3] G. Kriegsmann, A. Taflove, and K. Umashankar, "A New Formulation of Electromagnetic Wave Scattering Using an On-Surface Radiation Boundary Condition Approach," IEEE Trans. on Antennas and Prop., vol AP-35, No 2, February 1987, pp. $153-161$.

[4] O. Ramahi, A. Khebir, R. Mittra, "Numerically Derived Absorbing Boundary Condition for the Solution of Open Region Scattering Problems," IEEE Trans. on Antennas and Prop. , vol AP-39, No 3, March 1991, pp. 350-353. 


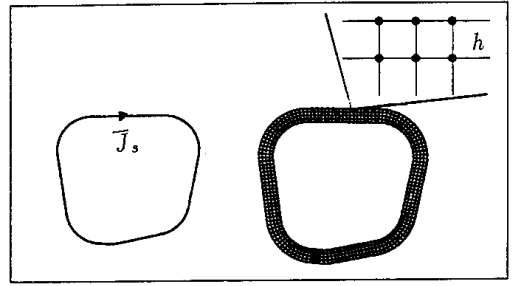

Figure 1: The object, the mesh around it, the metron currents, and the six points involved in the Measured Equation of Invariance.

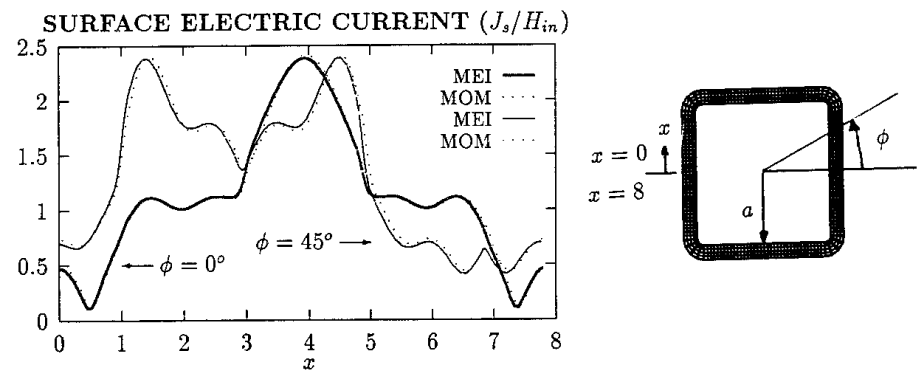

Figure 2: Currents on the surface of a square conducting cylinder illuminated by a TE plane wave incident from $\phi=0^{\circ}$ and $\phi=45^{\circ}$, compared to the method of moments $(\lambda=2, a=1)$.

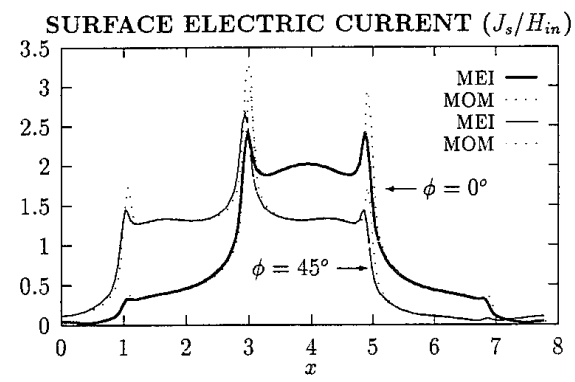

Figure 3: Currents on the surface of a square conducting cylinder illuminated by a TM plane wave incident from $\phi=0^{\circ}$ and $\phi=45^{\circ}$, compared to the method of moments $(\lambda=2, a=1)$. 
SURFACE ELECTRIC CURRENT $\left(J_{s} / H_{\text {in }}\right)$

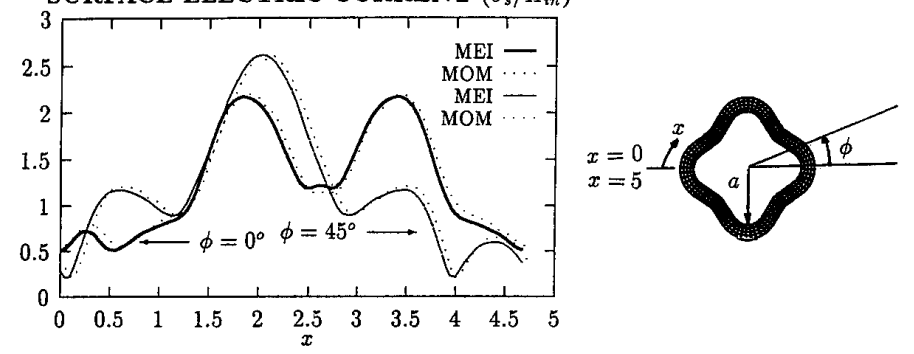

Figure 4: Currents on the surface of a concave conducting cylinder illuminated by a TE plane wave incident from $\phi=0^{\circ}$ and $\phi=45^{\circ}$, compared to the method of moments $(\lambda=2, a=0.8)$.

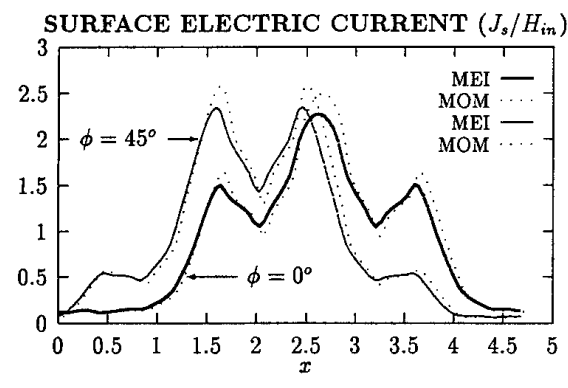

Figure 5: Currents on the surface of a concave conducting cylinder illuminated by a TM plane wave incident from $\phi=0^{\circ}$ and $\phi=45^{\circ}$, compared to the method of moments $(\lambda=2, a=0.8)$.

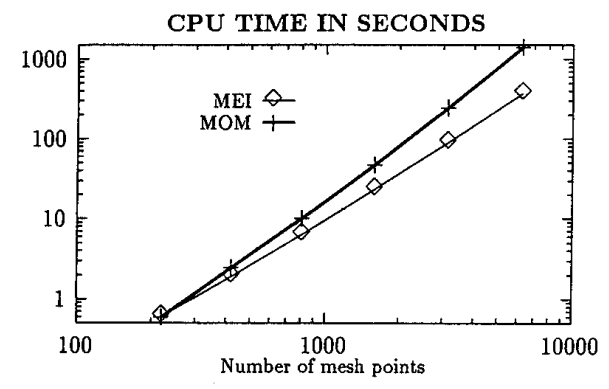

Figure 6: Comparison of the CPU time used by the MEI and MOM methods. 